\title{
Construcción socio-política de una vocación docente dirigida a la transformación social: el caso de la pedagogía en el campo de la educación física
}

\author{
Socio-political vocation construction toward social \\ transformation: Physical Education Teacher Teaching
}

*Ixa Andrea López Poblete

López, I. (2020). Construcción socio-política de una vocación docente dirigida a la transformación social: el caso de la pedagogía en el campo de la educación física. Revista Ciencias de la Actividad Física UCM, № 21(1), enerojunio, 1-14. DOI: http://doi.org/10.29035/rcaf.21.1.4

\begin{abstract}
RESUMEN
¿Tiene sentido hablar de vocación docente social y política en la formación universitaria de futuros profesores y profesoras de Pedagogía en el campo de la Educación Física? Es la interrogante que se discute en este artículo que defiende la necesidad y vigencia de esta cuestión, afirmando que la vocación docente también debe ser comprendida desde una perspectiva socio-política que allane, favorezca y aliente un proceso de reflexión, de modo alternativo, respecto de la conceptualización de la identidad profesional docente que, en la actualidad, aparece demarcada por prescripcionesy códigos normativos, al punto que se puede sostener que las políticas educativas han descuidado a la vocación como un concepto vinculado a la docencia. La dimensión socio-política de la vocación docente, formando parte de la identidad profesional, da lugar a una reflexión acerca de la formación inicial docente de profesores de Pedagogía en el campo de la Educación Física (EF) dentro de una contingencia marcada por la desigualdad y segregación social. Ello, además, va de la mano de estudios que plantean que la Pedagogía en Educación Física (PEF) conlleva una función transformadora de la sociedad que las políticas educativas no han tomado debidamente en cuenta. La teoría filosófica de Michel Foucault y la pedagogía de Paulo Freire, aunque desde perspectivas epistemológicas diferentes, permiten sostener el por qué en la actualidad se presenta como relevante y necesario volver a preguntarse cómo se construye una vocación docente social y política en la Pedagogía en el campo de la EF.
\end{abstract}

\section{Palabras clave}

Vocación, identidad, educación física, transformación social y justicia. 
López, I. (2020). Construcción socio-política de una vocación docente dirigida a la transformación social: el caso de la pedagogía en el campo de la educación física. Revista Ciencias de la Actividad Física UCM, N²1(1), enero-junio, 1-14. DOI: http://doi.org/10.29035/rcaf.21.1.4

\begin{abstract}
Does it make sense to talk about social and political vocation in Teacher Education in the Physical Education field? This question is discussed in order to preserve need and validity of this issue, stating that the teaching vocation must also be understood from a socio-political perspective to ease, favor and encourage a process of reflection, alternatively, regarding teaching professional identity conceptualization which nowadays, appears to be delimited by prescriptions and normative codes, at the stage that educational policies have neglected on conceptualizing vocation linked to teaching. Socio-political dimension of teaching vocation shaping professional identity, takes on account an uncertain environment characterized by inequality and social segregation, within the Physical Education Teacher Education field. In addition, this issue is unfolded together with research proposals of a social transformative function in the Physical Education Teacher Education context that educational policies have not duly taken into account. Michel Foucault's philosophical theory and Paulo Freire's pedagogy, although from different epistemological perspectives, allow us to sustain why at the present time it becomes relevant and necessary to ask us in which way socio-political teaching vocation is constructed in the field of Physical Education Teacher Education.
\end{abstract}

\title{
Key words
}

Vocation, identity, physical education, social transformation, social justice.

\section{Introducción}

La identidad profesional docente para una "buena" enseñanza es una temática de preocupación transversal en los programas de formación inicial docente (FID) cuyos resultados se basan en evidencias. La vocación, por su parte, aunque evitada en las políticas públicas, inevitablemente aparece vinculada la docencia. A su vez, la trayectoria formativa universitaria constituye un hito fundante para la construcción de la identidad profesional docente, las concepciones de docencia y el significado particular atribuido a la vocación docente, reconociendo ciertamente que también existen otras variables que influyen en esta configuración, tales como las biografías personales, las interacciones significativas, el sentido construido sobre las vivencias, entre otras.

El Ministerio de Educación (MINEDUC, 2013) por su parte, entrega a los establecimientos de educación superior lineamientos sobre el "buen" desempeño académico que dan cuenta de una identidad profesional docente circunscrita al desarrollo de competencias y habilidades que permiten rendir cuentas al Estado sobre la calidad en la educación en este ámbito. De tal forma que los programas formativos que guían la formación de aspirantes a profesor(a) en la carrera de pedagogía en el campo de la Educación Física y Salud, tal como se denomina hoy, entregan conocimientos conceptuales, procedimentales y actitudinales. Éstos tienen por objetivo entregar conocimientos disciplinares y pedagógicos como telón de fondo para conseguir ciertos niveles de rendimiento y actividad física, que entiende el bienestar humano en función de la salud física y una alimentación saludable. Por consiguiente, se anteponen competencias básicas controlables, pero se dejan de lado otras prioridades formativas que refuercen procesos democráticos y participativos, para "llegar a ser" individuos comprometidos con la sociedad y poseedores de una conciencia social que aporte a la formación y transformación de ciudadanos y, desde un saber-ser docente cuyas prácticas educativas permitan un desempeño en la complejidad de contextos de desigualdad e 
injusticia sociales, siendo capaces de actuar transformativamente en ellos.

Es llamativa la tendencia de matrícula total de carreras de pregrado de Pedagogía en Educación Física y similares que informa el Consejo Nacional de Educación (CNED, 2018) para el 2018 que asciende a un total de 11.575 matriculados. Número que se encuentra incluido en los índices que el CNED informa para ese mismo año en la totalidad de las carreras de pedagogía que alcanza a 48.988 matriculados. Bajo las condiciones contemporáneas de persistente desigualdad e injusticia social, esto no es un dato menor, si se toma en consideración que estos futuros profesores/as de EF ingresarán al campo laboral, formados desde una concepción en sintonía con la ideología neoliberal, que elude alternativas formativas para el desarrollo de una educación más justa y democrática.

En atención al estado de la contingencia política y social actual por la cual atraviesa Chile, surge como imperativo que las autoridades educativas tengan en consideración las desigualdades sociales existentes en un país donde sus índices son preocupantes, comparativamente, en el concierto latinoamericano (PNUD, 2017). Dado este escenario, parece necesario visibilizar el potencial de transformación social que tiene la Pedagogía en el campo de la EF, puesto que no figura como una asignatura comprometida con la justicia social ni tampoco asumiendo un rol político vinculado al contexto histórico de un sistema educativo (Moreno \& Arancibia, 2015). En consecuencia, la función de la pedagogía en EF en la actualidad nacional se encuentra descontextualizada de la realidad social y las políticas educativas. Tampoco se tiene en consideración la importante producción académica existente respecto del rol transformador de la EF, la cual va de la mano con una vocación docente dirigida a contribuir a las transformaciones sociales.

\section{Desarrollo}

\subsection{Identidad y vocación docente, en la trayectoria formativa universitaria de la Pedagogía en el campo de la EF}

Desde un enfoque histórico-cultural en Chile, se asume el planteamiento de Larraín (2001), para quien la identidad cultural es algo que está en permanente construcción y reconstrucción dentro de nuevos contextos y situaciones históricas. De tal forma que la construcción de la identidad es un proceso discursivo que no sólo mira el pasado como la reserva privilegiada donde están guardados los elementos principales de identidad, sino que también mira hacia el futuro y concibe a la identidad como un proyecto. La pregunta por la identidad es entonces no sólo ¿qué somos?, sino también ¿qué queremos ser?

Las identidades personales son formadas por identidades colectivas culturalmente definidas, pero éstas no pueden existir separadamente de los individuos señala Larraín (2001). Ahora bien, desde el punto de vista histórico en Chile (Núñez, 2007) la identidad docente ha transcurrido por distintos momentos, de suerte que han prevalecido visiones de la pedagogía como apostolado, el docente como funcionario público, el profesor como instructor (rol técnico) y la docencia entendida como profesión. En las últimas décadas ha cobrado mayor vigencia la representación del profesor como un profesional de la educación, que discursivamente reconoce al profesor como un intelectual reflexivo y transformativo que, paradojalmente, cuente con las competencias que correlacionen significativamente con el logro de aprendizajes medibles y cuantificables. La idea de reflexión crítica (metacognición orientada a la mejora de prácticas), entonces, es reducida a la dimensión técnica de la profesión.

Entre los requerimientos de evaluación ministeriales que prescriben la imagen del 
López, I. (2020). Construcción socio-política de una vocación docente dirigida a la transformación social: el caso de la pedagogía en el campo de la educación física. Revista Ciencias de la Actividad Física UCM, N²1(1), enero-junio, 1-14. DOI: http://doi.org/10.29035/rcaf.21.1.4

"buen" docente profesional, se encuentran los Estándares Orientadores para la Formación Inicial Docente (2013) (que llamaremos "estándares de egreso" o EE) para la carrera de Pedagogía en Educación Física y Salud, que proporciona orientaciones a las entidades de educación superior para la formación y la evaluación de saberes disciplinarios y pedagógicos mínimos al finalizar su formación profesional; el Marco para la Buena Enseñanza (MBE) (MINEDUC, 2008) como referencia para orientar el trabajo docente, mediante prescripciones sobre la evaluación de la "buena enseñanza" retratando a un profesional "efectivo" e ideal de la identidad profesional del docente; y la evaluación del proceso formativo según las "Bases curriculares para la pedagogía en educación física y salud en la enseñanza básica y media" (MINEDUC, 2019), que la destacan como una asignatura fundamental para el desarrollo integral de los estudiantes. Por consiguiente, al menos desde el punto de vista de la política educativa, la identidad profesional docente se construye sobre la base de estándares de aprendizajes que aluden a disposiciones y comportamientos virtuosos basados en competencias medibles para desempeñar efectivamente un rol de docente profesional.

En lo anterior encontramos una primera fuente de tensiones para plantear una vocación docente socio-política, que se complejiza cuando se constata la vigencia de representaciones del actor docente como "representante del estado nacional" y como trabajador con "vocación apostolar". Nociones ambas, dados los enmarcamientos de política, la construcción social de la docencia se convierte en una crisis de identidad (Cornejo, 2006 y 2008, Núñez, 2004 y 2007). Este proceso, señala Galáz (2011), es un equilibrio complejo e inestable porque la imagen de sí mismo del docente es balanceada en un contexto de asignaciones o atribuciones de lo que se debiera ser, hacer y saber, que de- signa una distancia entre una identidad docente real y una ideal.

Una segunda tensión surge entre la valoración de la identidad profesional docente, y la omisión de la vocación docente en el MBE. Mientras que la primera se asocia a habilidades y competencias profesionales, la segunda se encuentra intrínsecamente vinculada a la docencia. Desde una perspectiva psicológica se subentiende que el profesional de la docencia tiene vocación, y ésta constituye una dimensión de la identidad docente. No obstante, el constructo vocacional que acá se plantea, forma parte de un proceso de construcción social en evolución y cambio, producto de las interacciones sociales, vivencias y experiencias con sentido desde la biografía personal. Es decir, una subjetividad vinculada a la psicología individual, versus una subjetividad ligada a una dimensión discursiva y social.

De acuerdo a lo señalado anteriormente, el "buen" desempeño docente prescrito para la FID en la carrera de pedagogía en el campo de la EF responde al cumplimiento de competencias y habilidades para generar hábitos de vida activa y saludable en la comunidad y alcanzar menores niveles de obesidad y sedentarismo. Sin embargo, esta forma de entender la identidad profesional asume una racionalidad tecnocrática o pragmática que estandariza y homogeniza concepciones de enseñanza. Desde esta perspectiva, la identidad docente está ligada a una disposición del carácter como una dimensión individual y psicológica, que significa a la identidad como una entidad fija, finita, definida, prescrita y por tanto evaluable conceptual, procedimental o actitudinalmente, como una competencia y/o habilidad en el marco de las políticas ministeriales. Sin embargo, una educación instrumental como la descrita, muestra una cara de la moneda y oculta otra, de naturaleza social relacionada a una educación democrática dirigida a atender necesidades y 
López, I. (2020). Construcción socio-política de una vocación docente dirigida a la transformación social: el caso de la pedagogía en el campo de la educación física. Revista Ciencias de la Actividad Física UCM, № 21(1), enero-junio, 1-14. DOI: http://doi.org/10.29035/rcaf.21.1.4

transformaciones sociales, desde una concepción de proceso en lugar de resultados.

La problemática se complejiza cuando las políticas públicas, al dejar de lado la dimensión socio-política de la identidad profesional, restringen el sentido de agencia o autonomía docente y reproducen un círculo vicioso que obstaculiza el reconocimiento social formativo y transformativo de una pedagogía en el campo de la EF vinculada a la justicia social. La pedagogía en educación física, en consecuencia, es concebida y juzgada por su contribución a una vida sana y a indicadores antropométricos y de salud individual. Lo señalado anteriormente justifica problematizar la FID en cuanto a la hegemonía de una perspectiva biologicista y tecnocrática de la pedagogía en EF. En consecuencia, al omitir la dimensión socio cultural del cuerpo y la persona, se excluye una perspectiva holística del ser humano.

En contraste, acá se asume que la vocación docente socio-política conforma una dimensión de la identidad profesional docente, incluyente, complementaria, y factible de cohabitar con el "buen" desempeño que define el MBE. Desde una perspectiva filosófico-educativa se asume en este ensayo, una identidad docente siempre siendo, en constante recreación, un horizonte de exploración inacabado de búsqueda, que significa al mundo de múltiples maneras en tanto entidad situada socio-cultural e históricamente. Desde aquí surge la propuesta de una identidad vocacional socio-política que va más allá del "buen" desempeño docente definido por el MINEDUC en el MBE, ahora vinculada a un rol de los/as futuros profesores/as de EF con autonomía política desde el compromiso con el desarrollo de una educación más justa y democrática. En la práctica, significa una praxis situada en la contingencia para colocar sobre la mesa problemáticas sociales que afectan a la comunidad educativa, con libertad para manejar tensiones, para abrir espacios para el debate, y promover la contraposición de ideas.

\subsection{Una Pedagogía en el campo de la Educación Física para la transforma- ción social}

La formación inicial de la carrera de PEF evidencia planes de estudio que se caracterizan por ser heterogéneos debido a la existencia de una multidisciplinariedad de comprensiones acerca de la construcción de conocimiento, programas fragmentados y diversidad de enfoques epistemológicos frente a la concepción de cuerpo en movimiento, según la crítica de los autores (Portes, Almonacid \& Castro, 2014, Almonacid \& Moreno, 2012, Araya, 2006). Como resultado, las representaciones sociales de estudiantes de PEF siguen construyéndose en función del significado que le asignan a su especialidad la que vincula salud y bienestar al movimiento, y otorgan un valor pedagógico a la actividad física, y la recreación y el deporte (Caniuqueo et al., 2018). Los profesores de PEF en ejercicio de la profesión reproducen la cultura existente presentando incongruencias entre lo que se enuncia como positivo a desarrollar y lo que verdaderamente se aplica en la práctica cotidiana (Moreno, 2011), provocando el refuerzo de un modelo determinista y mecanicista que potencia lógicas de subordinación, pensamiento convergente y paramétrico (Toro, 2007).

Las políticas públicas, por su parte, priorizan una pedagogía en el campo de la EF con una acción motriz descontextualizada de un proceso de humanización (Portes, Almonacid \& Cáceres, 2014), no comprometida con la justicia social, persistente en la reproducción del statu quo de una profesión docente cuyo foco en el siglo XXI sigue puesto en el movimiento del cuerpo y en una actividad física basada en el rendimiento que invisibiliza una dimensión social y cultural. Por tanto, la representación social del futuro profesor/a de EF se reduce a una identidad 
López, I. (2020). Construcción socio-política de una vocación docente dirigida a la transformación social: el caso de la pedagogía en el campo de la educación física. Revista Ciencias de la Actividad Física UCM, N²1(1), enero-junio, 1-14. DOI: http://doi.org/10.29035/rcaf.21.1.4

profesional limitada por fuerzas sociales que desvalorizan esta carrera y a una autonomía relativa según disposiciones o comportamientos académicos medibles y evaluable por los EE del MINEDUC. Este círculo vicioso, a nuestro entender, actúa en desmedro de la construcción de un rol docente alternativo social e invisibiliza el potencial de transformación social de esta área curricular.

En sintonía, el cuerpo académico de la PEF ha colocado el acento en la tensión que resulta de cumplir con las prescripciones ministeriales en un escenario académico donde predomina una racionalidad tecnocrática en la PEF, además de bregar con las barreras burocráticas existentes. De allí que diversos autores señalan que cabría enfocar a esta carrera profesional de una manera distinta encaminada hacia una función transformadora de la sociedad y comprometida con la justicia social (Moreno \& Arancibia, 2015). La función de una PEF dirigida a transformar la sociedad, se encuentra planteada en los estudios de Moreno \& Medina, 2012, González, 2014, Moreno, Rivera, Trigueros \& Obando, 2012, Moreno, Gamboa \& Poblete, 2014a, Almonacid, 2010, Moreno, Gamboa \& Poblete, 2014b, Moreno, Rivera \& Trigueros, 2014, Moreno \& Poblete, 2015, Moreno \& Álvarez, 2010, Moreno, Trigueros \& Rivera, 2009, Pazos, Toro \& Sabogal, 2019, Toro \& Sabogal, 2018, Portes, Almonacid \& Castro, 2014, Toro \& Niebles, 2013, Almonacid \& Moreno, 2012, Almonacid, 2012, Tefarikis, 2006, Toro, 2007, 2010a y 2010b y Toro \& Valenzuela, 2014, entre otros. Todo este corpus bibliográfico justifica pensar que una función transformadora de la PEF va de la mano de una vocación docente atravesada por la justicia social, como dimensión de la identidad profesional en constante exploración, nunca acabada y comprometida con la construcción de una vida mejor para todos.

\subsection{Cómo se construye una vocación do- cente socio-política para la transfor- mación social}

Se asume el pensamiento filósofo-educativo de Michel Foucault (1926 - 1984) y la praxis pedagógica de Paulo Freire (1921-1997), quienes desde epistemologías diferentes permiten la contextualización de un sistema educativo en crisis como el chileno fundado en una ideología neoliberal y racionalidad tecnocrática. Foucault y Freire permiten discutir los efectos de la dominación, segregación y exclusión a partir de una conceptualización esencialista de la identidad profesional en función de la eficiencia como sinónimo de cumplir con competencias y habilidades predefinidas. En consecuencia, aparece una concepción que no deja espacio para pensar la educación de modo más cercano a la idea de comunidad, solidaridad y en sintonía con las nuevas transformaciones sociales.

Aunque divergentes en pensamiento, filosófico en Foucault, y fundamentado en la praxis en Freire, ambos autores permiten caracterizar significados profundos sobre las concepciones de sujeto-objeto, verdad, poder, libertad, liberación, política, educación, y cuerpo, entre otros, que pueden llegar a expresarse o manifestarse en un continuum desde formas de opresión, o como indicadores del camino hacia la liberación. El punto de encuentro es que para ambos la identidad es una construcción situada socio-cultural e históricamente en contextos que cambian y se transforman, pero difieren en la manera cómo se construye la identidad. Si bien para Foucault (1999 y 2012) es la identidad y no la vocación su preocupación, con Freire es posible analizar la dimensión socio-política de la vocación docente al significado filosófico-pedagógico de la identidad.

La construcción de la identidad, para Foucault, deviene desde la relación con- 
sigo mismo incluida en las relaciones de poder y en las relaciones de saber, y desde dimensiones discursivas y no discursivas que legitiman el conocimiento por regímenes de verdad. Foucault (2012) señala que la auto-construcción de identidad es ética en cuanto a que concibe al individuo dueño de su propia libertad frente a códigos y prescripciones como una práctica de libertad o autogobierno, como un proceso tanto social como individual que envuelve la deliberación sobre el cuidado de sí mismo y de los demás. La construcción de la identidad, para Freire, es praxis, es decir, la identidad se construye en tanto se materialice en relaciones intersubjetivas y dialógicas desde un lenguaje de posibilidad en búsqueda constante de recrear y reinventar nuevos conocimientos y verdades.

La centralidad que Foucault otorga al lenguaje hace sospechar de los discursos institucionalizados que retratan a una identidad del "buen" desempeño docente, y la ilusión de libertad de elección dentro de un orden discursivo que proviene del MBE que establece el MINEDUC. El cuidado de sí, o "trabajo ético" sobre la identidad que realiza el individuo consigo mismo a través de las "tecnologías del yo" (Foucault, 2012) en la práctica, se traduce en un proceso de autoconstrucción inacabado de un "yo" que para dejar de ser objeto y convertirse en profesor o profesora de EF, forma y/o transforma una concepción sobre el "buen" desempeño docente, como un ejercicio de libertad. Ello permite pensar sobre qué, cómo, y para qué es el sentido de autonomía dentro del marco prescriptivo que omite o desdibuja una noción socio-política, en la cotidianeidad de procesos de enseñanza-aprendizaje de los/ as futuros profesores/as de EF.

El pensamiento de Foucault permite plantear que hay posibilidades de libertad frente a la ilusión de autonomía, si se asume el reto político de hacer frente a la tensión que se produce y reproduce en un contexto de prácticas discursivas e ideológicas que encasillan y determinan una identidad profesional de los/as futuros profesores/as de PEF. Por el contrario, para Foucault, es la infinitud, el devenir y la constante creación y auto-construcción de la propia identidad y autonomía, aunque relativa, lo que posibilita ser dueño de la propia libertad para manejarse y negociar tensiones dentro de relaciones de poder y resistencia ante códigos y normativas prescriptivas.

Paulo Freire, por su parte, enmarca su pensamiento crítico en la Teología de la Liberación y los espacios políticos de izquierda, desde el cual los valores radicales de la iglesia dejan de tener un sentido de asistencialismo basado en la limosna privada y el paternalismo (así, por ejemplo, la concepción de caridad se transforma en responsabilidad social). El pensamiento crítico de Freire se fundamenta en la emancipación social para la liberación (Larraín, 2005), desde aquí se argumenta que una vocación docente para la transformación social es una dimensión de la identidad profesional docente, que se articula como un proceso de construcción social fundamentado en el compromiso con lograr transformaciones sociales y de la mano de un proceso emancipación. La propuesta de Freire se refiere a un lenguaje de posibilidad desde el encuentro dialógico y pedagógico para la co-construcción de mundos posibles. De tal forma que en este proceso es imprescindible que la reflexión y la acción se den simultáneamente, siendo la praxis fundamental en el proceso de formación de una conciencia crítica. Además, es constitutiva de la construcción social de la identidad (Freire, 1978).

El pensamiento de Foucault se entiende como una manera de liberarse de la sujeción desde el gobierno de sí mismo como un trabajo de auto-construcción ética, mientras que el pensamiento de Paulo Freire persigue la liberación desde relaciones intersubjetivas que posibilitan la construc- 
López, I. (2020). Construcción socio-política de una vocación docente dirigida a la transformación social: el caso de la pedagogía en el campo de la educación física. Revista Ciencias de la Actividad Física UCM, N²1(1), enero-junio, 1-14. DOI: http://doi.org/10.29035/rcaf.21.1.4

ción de conocimiento como un proceso en constante evolución y recreación, a través de lo más fundamental de su legado pedagógico, la praxis. En lo que respecta a la praxis desde una vocación socio-política, se relaciona a una identidad docente como toma de posición para negociar tensiones dentro de la complejidad y dinamismo de las relaciones de poder dentro del sistema educativo. El concepto de libertad que subyace al pensamiento de Freire es que "nadie libera a nadie, ni nadie se libera solo" (Freire, 1978). Cuando Freire señala que los hombres se liberan en comunión", hace referencia a que la verdad se construye socialmente por lo tanto es el saber de los sujetos (que dejan de ser objetos de opresión) lo que los hace libres. Ya que en el diálogo horizontal y colaborativo es donde las relaciones de poder se convierten en nuevos saberes para transformar visiones de mundo alternativas, diferentes, dinámicas y siempre cambiantes.

Foucault despierta la sospecha frente a los discursos de poder y Freire las posibilidades de transformación de discursos en forma inacabada. Ambos permiten sustentar y contextualizar en la pedagogía en el campo de la EF y realizar una crítica al sistema educativo chileno. profesores/as de pedagogía en el campo de la EF. Como consecuencia de una racionalidad instrumental predominante y mecanismos de estandarización del rendimiento tanto para estudiantes como profesores que atraviesa a todos los niveles educativos, hasta ahora no ha habido espacios para formar y transformar a los/as futuros profesores/as de EF con un sentido de autonomía e identidad de modo alternativo, cambiante e inacabado, que no se contradice con las competencias e identidades profesionales prescritas en el MBE que establece el MINEDUC. ¿Cómo se relaciona lo anterior con las concepciones de docencia y construcción vocacional de los/as futuros profesores/as de EF?

Los esfuerzos hasta antes de octubre de 2019 se asemejan a haber permanecido durante años bajo el alero del "síndrome de la desesperanza aprendida" (Larraín, 2001) y los chilenos a ser una nación acostumbrada al abuso, maltrato, segregación e injusticias Hoy la tarea es aprender de la historia para cambiar, entre otras cosas, también a un sistema educativo en crisis.

El imperio por la fuerza, durante la década de 1970, de una ideología neoliberal que valora la competitividad, el individualismo y las competencias, le impuso al sistema educativo una racionalidad tecnocrática basada en resultados estandarizados junto con un aprendizaje entendido como un fin en lugar de proceso. Además de cuestionable, desde distintos enfoques, no resulta sustentable, no solamente porque levanta concepciones de mundo como verdades universales y ahistóricas, sino porque además los mecanismos de rendición de cuentas en el sistema educativo han terminado socavando la equidad y democracia, al estructurar una educación en base a una concepción de la calidad educativa por cierto, injusta en sí misma. Esta particular visión sólo satisface los intereses de ciertos sectores, de tal forma que termina convirtiéndose en un
1. El estallido social en Chile en octubre,2019, despierta el descontento generalizado de una sociedad que se moviliza en reclamo por cambios urgentes ante la desigualdad, segregación e injusticias, reflejo de una profunda crisis social y política. 
sistema violento, al tiempo que refuerza positivamente a unos pocos y castiga, excluye, segrega, marginaliza al resto mayoritario; $y$, por ende, divide al país entre ricos y pobres, incluidos y excluidos, lo que en la práctica se traduce en la existencia de una buena educación versus una mala educación, entre igualdad y desigualdad, entre equidad e inequidad.

En el campo educativo, particularmente en la pedagogía en el campo de la EF, hoy aparece como necesario replantearse los programas formativos a partir de la cuestión de si el objetivo busca formar deportistas o, por el contrario, formar sujetos conscientes de su cuerpo, su salud ${ }^{2}$ que, a su vez, formen a ciudadanos en pos de la construcción de una sociedad más justa y mejor; y, desde este último objetivo, construir concepciones de docencia e identidades docentes vinculadas a una formación profesional en coherencia con principios cívicos de colaboración, solidaridad y justicia social, e histórico-culturalmente situada en pro de la integración social.

Las problemáticas sociales no escapan ni son ajenas a la pedagogía en el campo de la EF. En estos últimos decenios, probablemente, el "sentido común" Ilevó a no valorar esta área curricular en tanto disciplina pedagógica y terminó engendrando la reproducción de esquemas deportivizados de enseñanza. En el actual contexto social nacional, se da la oportunidad de abrir un debate permanente para desestabilizar y desafiar el sentido común y que la pedagogía en el campo de la EF recupere la importancia de una profesión valiosa en sí misma. El reto de aprender del pasado y mirar al futuro, como

2. En lugar de una concepción individual de la salud, hay otras miradas de la actividad física, como un encuentro posible con el otro estableciendo redes para fortalecer el cuidado de la propia salud como un hábito de vida. Mayor información se encuentra disponible en De Carvalho \& Soares, 2010. una forma de construir en forma inacabada una identidad alternativa de la pedagogía en el campo de la EF, con una proyección deportiva, social, política y pedagógica, se hace presente ahora. Si bien la discusión pudiese resultar desgastante e incómoda, redunda en una oportunidad histórica de carácter ético para revisar y abrir un debate autocrítico acerca la construcción y tipo de identidad de profesión docente deseada y necesaria para la sociedad.

La cuestión y discusión respecto de ampliar autocríticamente la mirada acerca del rol y función social que le cabe a la pedagogía en el campo de la EF queda abierta. ¿Formar profesores que formen a futuros deportistas que batan marcas históricas y destaquen en sus respectivas ramas? ¿O docentes que enseñen a enseñar con una vocación de compromiso por los cambios sociales en pos de una democracia participativa y sociedad más justa?

\section{Conclusiones}

En un momento histórico de fractura social como el actual, y a partir de un proceso de revisión autocrítico surge, entonces, como necesario volver a preguntarse acerca de cómo se conceptualiza y entiende a la vocación social y política de los/as futuros profesores/as de EF, ya que en lugar de pensarla de una manera que la des-profesionalice aludiendo la tradicionalmente mentada identidad apostolar, esta vez aparece afianzada sobre un fundamento social y político. Desde esta mirada de la PEF, entendida y concebida con un rol y función en pos del compromiso con transformaciones sociales, el concepto de vocación socio-política integrada a una dimensión de la identidad docente viene, entonces, a cobrar vigencia

Un ejemplo de una posición docentealumno en términos desiguales correspondería al de un docente formador de profesores de EF que enseña a sus estudiantes 
López, I. (2020). Construcción socio-política de una vocación docente dirigida a la transformación social: el caso de la pedagogía en el campo de la educación física. Revista Ciencias de la Actividad Física UCM, N²1(1), enero-junio, 1-14. DOI: http://doi.org/10.29035/rcaf.21.1.4

a ser deportistas. Opuesto y deseable sería que los futuros profesores aprendan a enseñar a sus alumnos, no solamente a realizar técnicamente bien un deporte determinado, sino que desarrollen propias destrezas y conocimientos que les permitan apropiarse de convicciones sobre "qué tipo de sociedad quieren para Chile" desde una perspectiva colaborativa, participativa, es decir, también principios que formen parte de su identidad vocacional docente social y política, que se traduce en la infinita predisposición a inventar formas diferentes de pensar y actuar sobre el sí mismo en relación con la profesión docente, sobre todo en aquello que pudiese atentar contra la dignidad humana o pudiera constituir una limitación a las posibilidades de formar y transformar a los/as futuros profesores/as de EF orientados y comprometidos, con aportar en la construcción de un país desde la solidaridad y la vida en comunidad.

Una identidad profesional docente fundamentada en competencias y habilidades para el "buen" desempeño docente, tal como una PEF estandarizada, no solo omite al cuerpo como una construcción social y cultural, sino que también limita el sentido de agencia, ya que neutraliza el potencial socio-político de los/as futuros profesores/as de EF. Al esencialismo que reduce la identidad a una entidad estática y predeterminada, desde una racionalidad tecnocrática, se propone un modo alternativo de entender a la identidad profesional docente y la vocación socio-política como una dimensión de ésta.

Una FID encargada de formar estudiantes de PEF para intervenir en la sociedad para transformarla, representa una praxis inacabada de acciones pedagógicas para prevenir situaciones de injusticia, en lugar de sólo reaccionar a la contingencia. En la praxis freireana, significa ocuparse y preocuparse permanentemente sobre situaciones de discriminación tales como: clase social, etnia, capacidad, incapacidad o capacidades diferentes para realizar actividad física, concepción de normalidad sobre masculinidad o femineidad, prejuicios o estereotipos que pudieran estigmatizar cuerpos, entre otras temáticas atravesadas por criterios de justicia social. La vocación docente desde una perspectiva socio-política y como una dimensión de la identidad profesional se construye día a día, siempre en proceso de "llegar a convertirse" o responder a la pregunta "¿qué profesor o profesora de EF quiero llegar a ser?". Sería engañoso pensar la vocación docente como algo con lo cual se nace o se construye sola a través de charlas, seminarios o cursos on-line.

Por otra parte, las innovaciones curriculares con un sentido de compromiso social se traducen en considerar al currículum formativo como un espacio de resistencia, discusión y construcción de identidades docentes. En un continuum, desde una actitud de alerta frente a dogmatismos, tal como una identidad vocacional docente con una sustancia ética que universalice o estandarice el conocimiento, hasta una actitud de apertura a una sustancia ética sobre la emancipación como proceso y en lugar de un fin. Ya que los extremos opuestos no solo restan la libertad de pensar a la identidad profesional docente de manera alternativa, sino que se corre el riesgo de reproducir un statu quo también alternativo. La invitación es a sospechar permanentemente de los discursos que, sin darnos cuenta, se han ido convirtiendo en sentido común.

En la práctica, y particularmente en pedagogía en el campo de la EF, se relaciona con la evidencia de vivencias de práctica profesional sobre el manejo tensiones de situaciones de discriminación o cualquier forma de injusticia o dominación, con mentores comprometidos con la justicia social. Con el objetivo de que el "buen" desempeño profesional en lugar de un punto de término, sea un proceso inacabado de construcción 
con conciencia social, la autonomía formando parte de la identidad vocacional docente desde una praxis profesional participativa, para construir sentido de pertenencia en una sociedad que en Chile se encuentra desprovista de una visión integradora.

En concordancia con lo señalado anteriormente, se concluye que tiene sentido hablar de vocación docente socio-política en la formación universitaria de los/as futuros profesores/as de Pedagogía en el campo de la Educación Física.

\section{REFERENCIAS BIBLIOGRÁFICAS}

Almonacid, A. (2012). La educación física como espacio de transformación social y educativa: perspectivas desde los imaginarios sociales y la ciencia de la motricidad humana. Estudios Pedagógicos XXXVIII, (Número Especial 7), 177-190. Recuperado de https://www.redalyc.org/ pdf/1735/173525520004.pdf

Almonacid, A. (2010). Aproximaciones a un estado del arte en la formación de profesores de educación física. Puntuaciones y reflexiones posibles. Motricidad y Persona, 7, 17-25. DOI: 10.5027/jmh-Vol13-Issue2(2012)art56

Almonacid, A. \& Moreno, A. (2012). Perspectivas epistemológicas en la formación del Profesorado de Educación Física: Sustento Teórico y Directrices para la Práctica Educativa. Motricidad Humana 13(2), 93-103. DOI: 10.5027/jmhVol13-Issue2(2012)art56

Araya, E. (2006). ¿Qué educación física para la escuela hoy?. Pensamiento Educativo. Revista de Investigación Educacional Latinoamericana, 38(1), 172-185. Recuperado de http://pensamientoeducativo.uc.cl/index.php/pel/ article/view/309/666
Caniuqueo, A., Hernández-Mosqueira, C., Troyano, A. Riquelme, D., Vargas, A., Vargas, R. \& Fernandes, J. (2018). Representaciones sociales: el significado de la educación física para los estudiantes de esa disciplina. Revista Electrónica de Investigación Educativa, 20(1), 104-111. Recuperado de https://doi.org/10.24320/redie.2018.20.1.1497

CNED, Consejo Nacional de Educación. (2018). Tendencia de matrícula total Pedagogía en Educación Física y similares por institución. Recuperado de https://www.cned.cl/indices/matricula-institucionesprogramas-educacion-superior

Cornejo, R. (2008). Entre el sufrimiento individual y el sentido colectivo: Salud laboral docente y condiciones de trabajo. Revista Docencia, 35, 77-85. Recuperado de https://trabajo-docente. webnode.cl/news/entre-el-sufrimiento-individual-y-los-sentidos-colectivos-salud-laboral-docente-y-condiciones-de-trabajo/

Cornejo, R. (2006). El trabajo docente en la institución escolar. La apropiaciónenajenación del proceso de trabajo docente en el contexto de las reformas educativas neoliberales.Revista de Psicología, 15(2), 9-27. DOl: 10.5354/0719-0581.2012.18390

De Carvalho, Y. \& Soares, F. (2010). Práticas corporais e grande saúde: um encontro possivel. Revista Movimento, Porto Alegre, 16(4), 229-244. Recuperado de http://producao.usp.br/handle/ BDPI/14616

Freire, P. (1978). Pedagogía del oprimido. Madrid: Siglo veintiuno editores. 
López, I. (2020). Construcción socio-política de una vocación docente dirigida a la transformación social: el caso de la pedagogía en el campo de la educación física. Revista Ciencias de la Actividad Física UCM, N²1(1), enero-junio, 1-14. DOI: http://doi.org/10.29035/rcaf.21.1.4

Foucault, M. (2012). Historia de la sexualidad 2: el uso de los placeres. Madrid: Biblioteca Nueva.

Foucault, M. (1999). Tecnologías del yo y otros escritos afines. Barcelona: Paidós Ibérica S.A.

Galaz, A. (2011). El profesor y su identidad profesional: ¿facilitadores u obstáculos del cambio educativo?. Estudios pedagógicos (Valdivia), 37(2), 89-107. Recuperado de http://dx.doi.org/10.4067/ s0718-07052011000200005

González, L. (2014). Representaciones sociales de los y las estudiantes de pedagogía sobre la educación física escolar en Chile (Tesis doctoral). Universidad de Granada, España. Recuperado de https://hera.ugr.es/tesisugr/24328509.pdf

Larraín, J. (2001). La identidad chilena. Santiago de Chile: LOM Ediciones.

Larraín, R. (2005). La naturaleza de la crítica: Teoría crítica y Paulo Freire. Paulo Freire. Revista de Pedagogía Crítica (3), 103-116. Recuperado de https://doi. org/19.25074//07195532.3.513

Ministerio de Educación de Chile, MINEDUC (2019). Bases curriculares para la carrera de Pedagogía en educación física y salud para la enseñanza básica y media. Unidad de Currículum y Evaluación. Ministerio de Educación de Chile. Recuperado de https://www. curriculumnacional.cl/614/w3-propertyvalue-120094.html

Ministerio de Educación de Chile, MINEDUC (2013). Estándares orientadores para las carreras de pedagogía en Educación Física. Santiago. Ministerio de Educación de Chile. Recuperado de https://www.cpeip.cl/wp-content/ uploads/2019/03/Est\%C3\%Alndares_ Educacion_fisica-2.pdf
Ministerio de Educación de Chile, MINEDUC (2008). Marco para la buena enseñanza. Ministerio de Educación de Chile. Recuperado de https://www. docentemas.cl/docs/MBE2008.pdf

Moreno, A. (2011). Percepciones del profesorado universitario de educación física en relación a la función de transformación social de la educación física escolar: el caso de Chile. (Tesis doctoral). Universidad de Granada, España.

Moreno, A., Rivera, E., Trigueros, C. \& Obando, V. (2012). Percepción de profesores universitarios chilenos sobre las funciones de la educación física escolar. Multidisciplinary Journal of Educational Research, 2(2), 202-227. DOI: 10.4471/remie.2012.10

Moreno, A. \& Poblete C. (2015). La educación física chilena y su profesorado: proponiendo algunos retos para la investigación en el área, Retos, 28, 291-296. Recuperado de www.retos.org

Moreno, A., Gamboa, R. \& Poblete, C. (2014a). La educación física en Chile: análisis crítico de la documentación ministerial. Revista Brasileña de Ciencias del Deporte, 36(2), 417-427. Recuperado de http://www.scielo.br/pdf/rbce/ v36n2/0101-3289-rbce-36-02-00411. pdf

Moreno, A., Gamboa, R. \& Poblete, C. (2014b). Desigualdad, educación y posibilidades de transformación social: Pedagogía crítica y democratización escolar. En A. Moreno y M. Arancibia (Eds.). Educación, Escuela y Transformación Social. Construyendo una ciudadanía crítica (pp. 11-21). Valparaíso: Colecciones EUV. 
Moreno, A., Rivera, E. \& Trigueros, C. (2014). Sistema de medición de la calidad de la educación física chilena. Revista Movimento, 20(1), 145-167. DOI: $10.22456 / 1982-8918.41485$

Moreno, A., Trigueros C. \& Rivera E. (2009). Pedagogía Crítica formación de profesores y educación física. Una revisión preliminar. En W. Moreno \& S. Pulido (Cord.). Universidad, currículo y educación física (pp. 37-52). Colombia: Universidad de Antioquia. Recuperado de http://viref.udea.edu.co/ contenido/publicaciones/expo2009/ curriculo_2009.pdf

Moreno, A. \& Arancibia, M. (2015). Educación, Escuela y Transformación Social. Construyendo una ciudadanía crítica. Valparaíso: Colecciones EUV.

Moreno, A. \& Álvarez, N. (2010). Creencias del profesorado universitario de Educación Física en relación a los conceptos de cuerpo y salud. Estudios pedagógicos (Valdivia), 36(1), 159-175. Recuperado de https://dx.doi.org/10.4067/ s0718-07052010000100009

Moreno, A. \& Medina, J.M. (2012). Escuela, educación física y transformación social. Estudios pedagógicos (Valdivia), 38(especial), 7-11. https://dx.doi.org/10.4067/s071807052012000400001

Núñez, I. (2004). La identidad de los docentes: una mirada histórica en Chile (Documento PIIE). Santiago, Chile: Programa Interdisciplinario de Investigaciones en Educación. Recuperado de http://identidadocente. uc.cl/documentos/6-nunez-2004
Núñez, I. (2007). La profesión docente en Chile. Saberes e identidades en su historia. Pensamiento Educativo, 47(2), 149-164. Recuperado de https:// pensamientoeducativo.uc.cl/files/ journals/2/articles/421/public/421-9381-PB.pdf

Pazos, J.M., Toro, S. \& Sabogal, A. (2019). Motricidad, educación y contexto: la dimensión política de la motricidad. Revista Internacional d'Humanitats 46/47. Recuperado de http://www. hottopos.com/rih 46/6\%20-\%20 JOSe\%20PAZOS.pdf

PNUD (2017). Desiguales. Orígenes, cambios y desafíos de la brecha social en Chile. Santiago de Chile, Programa de las Naciones Unidas para el Desarrollo. Recuperado de https://www.undp. org/content/dam/chile/docs/pobreza/ undp_cl_pobreza-Libro-DESIGUALES-final.pdf

Portes, J.M., Almonacid, A. \& Castro, J.C. (2014). Reflexiones respecto de un plan de estudio para pedagogía en educación física con fundamentación paradigmática en la ciencia de la motricidad humana. Motricidad Humana 15(2), 123-133. Recuperado de https://dialnet.unirioja.es/servlet/ articulo?codigo $=6348122$

Tefarikis, E. (2006). Motricidad humana, un cambio urgente y necesario. Pensamiento Educativo, 38, 94-107. Recuperado de http://www.pensamientoeducativo.uc.cl/files/journals/2/ articles/304/public/304-709-7-PB.pdf

Toro, S. \& Sabogal, A. (2018). Sentidos de la motricidad en Abya Yala. Revista Tandem, Didáctica de la Educación Física, 59, 8-13. 
Toro, S. \& Valenzuela, P. (2014). Educación para y desde la militancia y la transformación social: Nuevas racionalidades didácticas. En A. Moreno y M. Arancibia (Eds.). Educación, Escuela y Transformación Social: Construyendo una ciudadanía crítica. (pp. 54-65). Valparaíso: Colecciones EUV.

Toro, S. \& Niebles, A. (2013). Corporeidad y aprendizaje en el contexto de la Enseñanza General Básica: comprensión y descripción de los procesos de construcción de conocimiento desde la acción relacional de los actores. Estudios Pedagógicos, 39(1), 269-284. Recuperado de http://dx.doi.org/10.4067/ s0718-07052013000100016.

Toro, S. (2007). Una aproximación epistemológica a la didáctica de la motricidad desde el discurso y la práctica docente. Estudios pedagógicos (Valdivia), 33(1), 29-43. Recuperado de https://dx.doi.org/10.4067/S071807052007000100002

Toro, S. (2010a). Corporeidad y Lenguaje: La acción como texto y expresión. Cinta Moebio 37, 44-60. Recuperado de http://dx.doi.org/10.4067/s0717554X2010000100004
Toro, S. (2010b). Neurociencias y Aprendizaje... texto en construcción. Estudios pedagógicos (Valdivia), 36(2), 313-331. Recuperado de https://dx.doi.org/10.4067/s071807052010000200018

\section{Dirección para correspondencia}

Ixa Andrea López Poblete

Magíster Psicología de la Actividad Física y el Deporte. Doctorado en Educación.

Universidad Academia Humanismo Cristiano. Santiago, Chile.

Dirección Postal: Santa Julia 948, Ñuñoa, Santiago. Región Metropolitana, Chile.

Código postal: 7760516

ORCID: https://orcid.org/0000-0002-0631-425X

Contacto:

ixandrea.lopez@gmail.com

Recibido: 17-12-2019

Aceptado: 19-03-2020 